\title{
A Study on Physical Performance for Poly(L-lactic acid) in Addition to Layered Strontium Phenylphosphonate
}

\author{
Yan-Hua Zhang and Yan-Hua Cai \\ Chongqing Key Laboratory of Environmental Materials \& Remediation Technologies, \\ Chongqing University of Arts and Sciences, Chongqing 402160, China
}

Correspondence should be addressed to Yan-Hua Cai; caiyh651@aliyun.com

Received 5 March 2016; Revised 18 April 2016; Accepted 20 April 2016

Academic Editor: Hideto Tsuji

Copyright ( 2016 Y.-H. Zhang and Y.-H. Cai. This is an open access article distributed under the Creative Commons Attribution License, which permits unrestricted use, distribution, and reproduction in any medium, provided the original work is properly cited.

\begin{abstract}
The organic-inorganic hybrid layered strontium phenylphosphonate ( $\mathrm{SrP}$ ) was synthesized by using strontium chloride and phenylphosphinic acid. And the influence of layered SrP on the crystallization behavior and thermal stability of poly(L-lactic acid) (PLLA) was investigated through DSC, XRD, and TGA. Both DSC and XRD results demonstrated that layered SrP had the powerful accelerated ability for PLLA crystallization, and in the range of studied concentration, $0.7 \mathrm{wt} \%-1 \mathrm{wt} \%$ is the optimum concentration range to achieve rapid crystallization of PLLA. Meantime, as a result, the increase of cooling rate in nonisothermal crystallization procedure seriously affected the crystallization accelerated efficiency of SrP. Thermal stability measurement showed that layered SrP could cause the onset decomposition temperature of PLLA to decrease, but the thermal decomposition behavior of PLLA hardly depended on the SrP concentration.
\end{abstract}

\section{Introduction}

The metal phenylphosphonate is one of the most important synthesized inorganic/organic hybrid materials. According to its unique structure similar to layered clay [1], metal phenylphosphonate has attracted continuous growing attentions in recent years [2]. Thus, many works based on metal phenylphosphonate were explored to investigate its function and effect. In chemical synthesis field, the flower-like porous aluminum phenylphosphonate microspheres with an average diameter of $8 \mu \mathrm{m}$ were reported, and this flower-like porous structure could be achieved through a hydrothermal method; the relevant experimental results showed that the nanoflakes appearing in the initial growing stage exhibited a very important role in the formation of microflowers structure [3], whereas the aluminum phenylphosphonate was also obtained by refluxing reaction in water, and the resultant aluminum phenylphosphonate exhibited a layered structure with an interlayer spacing about $15 \AA$ [4]. Mahmoudkhani and Langer [5] reported that the synthesized calcium phenylphosphonate not only had excellent thermal stability, but also was not soluble in water and organic solvents upon $\mathrm{pH}$ higher than 2 , indicating a promising application in surface treatment field. In functional additive field, layered zinc phenylphosphonate was used to serve as nucleating agent for isotactic polypropylene. The crystallization behavior study showed that $0.1 \mathrm{wt} \%$ layered zinc phenylphosphonate caused the crystallization half-time of polypropylene to decrease from 19.42 to $1.87 \mathrm{~min}$ at $134^{\circ} \mathrm{C}$ due to the increase of nuclei density in polypropylene matrix. However, zinc phenylphosphonate could not change the crystalline structure of polypropylene [1]. Similarly, zinc phenylphosphonate was also found to be a good nucleating agent on the crystallization of the as-bacterially synthesized poly(3-hydroxybutyrate) and poly[(3-hydroxybutyrate)-co(3-hydroxyhexanoate)] [6] and poly(vinylidene fluoride) [7]. Additionally, the layered metal phenylphosphonate could also play an important role in enhancing the thermal stability and mechanical performance of polymers; the literature [8] reported that the layered lanthanum phenylphosphonate could remarkably promote the thermal decomposition temperature, the tensile strength, and storage moduli of glassfiber reinforced poly(ethylene terephthalate)/microencapsulated red phosphorus systems. In catalysis field, He et al. 
[9] firstly synthesized the zirconium phenylphosphonateanchored methyltrioxorhenium, and then the evaluation of the catalytic performance for epoxidation of cyclohexene was performed. The relevant results suggested that zirconium phenylphosphonate-anchored methyltrioxorhenium had advanced catalytic activity and selectivity for cyclohexene epoxidation.

Poly(L-lactic acid) (PLLA) is type of environmentfriendly polymer materials owing to its biodegradability and green production technology. Especially with the worse environmental pollution day by day, PLLA will become important general-purpose plastics and be employed in more industry and daily life fields. However, so far there are still some challenges, such as slow crystallization rate and poor heat resistance [10], to restrict the development of PLLA industry. In the case of application, it is a fact that improvement of PLLA crystallization rate is very critical. Herein, many attempts were carried out to overcome this defect [11-13]. For example, a small amount of both multiwalled carbon nanotubes and graphene nanosheets could accelerate the overall crystallization of PLLA, but the multiwalled carbon nanotubes with above $2 \mathrm{wt} \%$ concentration almost unchanged the overall crystallization rate of PLLA, and a high amount of graphene nanosheets (above $2.5 \mathrm{wt} \%)$ even retarded crystallization rate, resulting from the network structures of multiwalled carbon nanotubes and graphene nanosheets [14]. The zinc lactate is also an effective additive for changing PLLA crystallization behavior; the result of isothermal crystallization measurement showed that the crystallization half-time of $7 \mathrm{wt} \%$ zinc lactate nucleated PLLA decreased from $2359.91 \mathrm{~s}$ to $152.68 \mathrm{~s}$ at $115^{\circ} \mathrm{C}$ [15].

Recently, the metal phenylphosphonate was also introduced into PLLA matrix to promote its crystallization performance [16-18]. Pan et al. [17] reported that the addition of $0.02 \mathrm{wt} \%$ layered zinc phenylphosphonate could make PLLA finish crystallization at high cooling rate $\left(10^{\circ} \mathrm{C} / \mathrm{min}\right)$. Furthermore, with further increasing of zinc phenylphosphonate concentration, the crystallization rate also increased. The comparative study on nucleating ability of three metal phenylphosphonates including zinc phenylphosphonate, barium phenylphosphonate, and calcium phenylphosphonate was investigated by Wang and his colleagues [18]. And the nucleating ability for PLLA was as the following order: zinc phenylphosphonate $>$ calcium phenylphosphonate $>$ barium phenylphosphonate. However, as far as we know, there is no report of the effect of layered strontium phenylphosphonate on the performance of PLLA. Meantime, it is very necessary to develop more metal phenylphosphonates to confirm the nucleation mechanism of metal phenylphosphonate for PLLA. Thus, in this work, the layered strontium phenylphosphonate was synthesized through phenylphosphinic acid and strontium chloride; then the crystallization behavior and thermal stability of PLLA incorporation of the layered strontium phenylphosphonate were investigated using DSC, $\mathrm{XRD}$, and TGA. This work is of interest to develop more PLLA composites modified by inorganic/organic hybrid material.

\section{Experimental Section}

2.1. Materials. Poly(L-lactic acid) (2002D) was purchased from Nature Works LLC, USA. The phenylphosphinic acid was obtained from Shanghai Danxi Chemical Science \& Technology Co., Ltd., China. The strontium chloride and sodium hydroxide were supplied by Chongqing Chuandong Chemical (Group) Co., Ltd., China.

2.2. Synthesis of Strontium Phenylphosphonate (SrP). The phenylphosphinic acid was dissolved in water with stirring, the strontium chloride aqueous solution was slowly added into the phenylphosphinic acid solution, and then $\mathrm{pH}$ of the mixture solution was adjusted to $5-6$ using $0.1 \mathrm{~mol} / \mathrm{L}$ sodium hydroxide. The mixture solution was filtrated, and the filtrate was stirred at $50^{\circ} \mathrm{C}$ to achieve the full crystallization, followed by filtrating again. The resultant product was washed by methanol and deionized water for 3 times, respectively. Finally, the strontium phenylphosphonate $(\mathrm{SrP})$ was dried in vacuum at $45^{\circ} \mathrm{C}$.

2.3. Preparation of PLLA/SrP Sample. The preparation of PLLA modified by SrP was performed in torque rheometer, and the melt processed procedure is the following: PLLA and $\mathrm{SrP}$ were blended with a rotation speed of $32 \mathrm{rpm}$ for $7 \mathrm{~min}$ at $180^{\circ} \mathrm{C}$ and at $64 \mathrm{rpm}$ for $7 \mathrm{~min}$ at $180^{\circ} \mathrm{C}$. Then the PLLA/SrP samples were hot pressed and cool pressed for $7 \mathrm{~min}$, respectively.

\subsection{Testing}

2.4.1. Differential Scanning Calorimeter (DSC). The nonisothermal crystallization behaviors of the neat PLLA and PLLA/SrP samples were measured through differential scanning calorimeter (Q2000, TA instrument, USA). The measured samples were firstly heated to the set melting temperature $\left(180^{\circ} \mathrm{C}\right)$ for $3 \mathrm{~min}$ and then cooled from $180^{\circ} \mathrm{C}$ to $40^{\circ} \mathrm{C}$ at different cooling rate. Finally, the samples were heated to $180^{\circ} \mathrm{C}$ again at a heating rate of $10^{\circ} \mathrm{C} / \mathrm{min}$, and the relevant nonisothermal crystallization data were recorded to further analysis.

2.4.2. X-Ray Diffraction (XRD). XRD measurements of layered SrP and PLLA/SrP samples after different conditions were operated on a diffractometer (XRD6000, Shimadzu, Japan) at room temperature with scanning rate of $2^{\circ} / \mathrm{min}$.

2.4.3. Thermogravimetric Analysis (TGA). The thermal stability of the SrP, PLLA, and PLLA with different concentration of $\mathrm{SrP}$ was recorded using a thermal analyzer (Q500, TA instrument, USA) under $60 \mathrm{~mL} / \mathrm{min}$ air flow from room temperature to $650^{\circ} \mathrm{C}$.

2.4.4. Scanning Electron Microscopy (SEM). The morphology of the layered $\mathrm{SrP}$ was observed by scanning electron microscopy (XL-30 ESEM FEG, Philips, Holland) in 15$20 \mathrm{kV}$ accelerating voltage (Tungsten filament). 


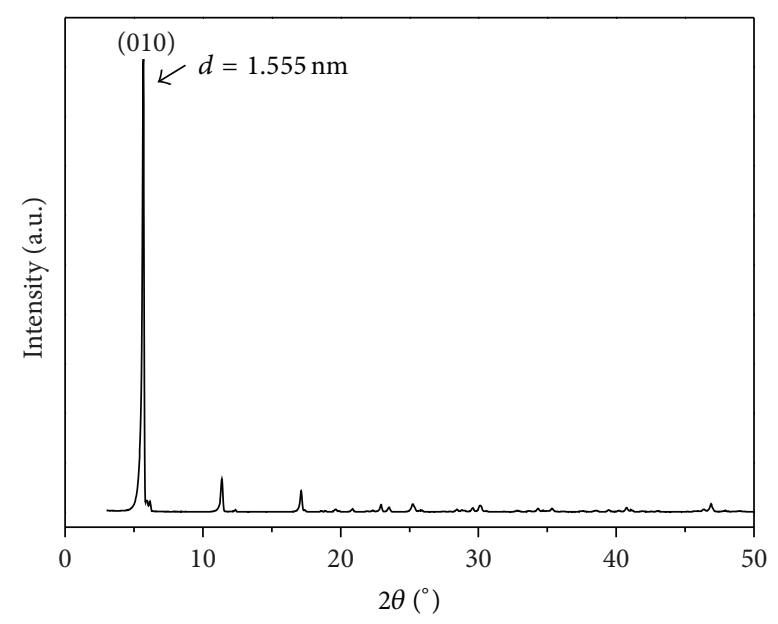

FIGURE 1: XRD of SrP.

\section{Results and Discussion}

3.1. Structure of SrP. Figure 1 presents the XRD pattern of aforementioned synthesized SrP. It is easily found that the $\mathrm{XRD}$ pattern of $\mathrm{SrP}$ is in good agreement with reported result [19], demonstrating the successful preparation of SrP. And the characteristic and strongest diffraction peak of $\mathrm{SrP}$ in Figure 1 appears at $2 \theta=5.68^{\circ}$, belonging to the $(010)$ plane reflection. The relevant interlayer spacing $d_{010}$ of $\mathrm{SrP}$ is $1.555 \mathrm{~nm}$, indicating that the phenyl exists in the interlamellar region beyond that of the phosphate group [17].

The study of the morphology is of great importance to understand the structure arrangement of SrP and evaluate the effect of SrP on performance of the primary PLLA. Therefore, the morphology of $\mathrm{SrP}$ was observed through SEM. As shown in Figures 2(a) and 2(b), the SrP exhibits type layered structure similar to layered silicates and other layered metal phenylphosphonate including lanthanum phenylphosphonate [8], calcium phenylphosphonate [5], and barium phenylphosphonate [20]. And it is also clearly observed from the enlarged images (Figures 2(c) and 2(d)) that this layered structure is formed by many lamellae with thickness of about $6 \mathrm{~nm}$, and these lamellae are in favor of promoting the crystallization of the pristine PLLA [21]. Besides, the high decomposition temperature of $\mathrm{SrP}$ in the following text further suggests the possibility of SrP for serving as nucleating agent for accelerating the crystallization of PLLA.

3.2. Nonisothermal Crystallization. The heat resistance and practical process of semicrystalline polymer primarily depend on the crystallization behavior. In particular, the study of nonisothermal crystallization behavior is more valuable for practical application. Figure 3 is the nonisothermal crystallization curves of the pristine PLLA and PLLA with different concentration of SrP from melt at a cooling rate of $1^{\circ} \mathrm{C} / \mathrm{min}$. As for the pristine PLLA, the crystallization peak is negligible under this nonisothermal crystallization condition owing to the poor crystallization ability. However, in the case of all PLLA/SrP samples, the sharp nonisothermal crystallization peaks appear in
TABLE 1: DSC data of PLLA and PLLA/SrP samples at a cooling rate of $1^{\circ} \mathrm{C} / \mathrm{min}$.

\begin{tabular}{lccccc}
\hline Sample & Rate $/{ }^{\circ} \mathrm{C} / \mathrm{min}$ & $T_{o} /{ }^{\circ} \mathrm{C}$ & $T_{\mathrm{cp}}{ }^{\circ} \mathrm{C}$ & $\Delta H_{c} / \mathrm{J} \cdot \mathrm{g}^{-1}$ & $T_{m} /{ }^{\circ} \mathrm{C}$ \\
\hline PLLA & 1 & 101.4 & 94.5 & 0.1 & - \\
\hline PLLA/0.3\% SrP & 1 & 111.6 & 107.1 & 30.7 & 145.8 \\
PLLA/0.7\% SrP & 1 & 116.3 & 109.4 & 31.0 & 146.2 \\
PLLA/1\% SrP & 1 & 117.1 & 110.1 & 30.6 & 146.9 \\
PLLA/1.5\% SrP & 1 & 116.9 & 110.1 & 30.4 & 146.8 \\
\hline
\end{tabular}

nonisothermal crystallization procedure, indicating the advanced crystallization accelerated ability of SrP for PLLA. On the other hand, it is a distinct fact that the effect of SrP concentration on the nonisothermal crystallization is very weak through the DSC data analysis (see Table 1). When SrP concentration is from $0.3 \mathrm{wt} \%$ to $1.5 \mathrm{wt} \%$, the onset crystallization temperature $\left(T_{o}\right)$, the crystallization peak temperature $\left(T_{\mathrm{cp}}\right)$, and the nonisothermal crystallization enthalpy $\left(\Delta H_{c}\right)$ exhibit slight increase, and at $1 \mathrm{wt} \%$ loading of the layered $\mathrm{SrP}$, the $T_{o}$ and $T_{\mathrm{cp}}$ have the largest value of $117.1^{\circ} \mathrm{C}$ and $110.1^{\circ} \mathrm{C}$, respectively. But $0.7 \mathrm{wt} \%$ SrP makes the PLLA obtain the largest $\Delta H_{c} 31.0 \mathrm{~J} \cdot \mathrm{g}^{-1}$. This slight effect of the SrP concentration on the crystallization behavior of PLLA shows that a small amount of SrP can achieve the dramatic enhancement of PLLA crystallization rate, indicating that layered $\mathrm{SrP}$ is a potential candidate for use in rapid prototyping modified PLLA materials. In addition, because the pristine PLLA almost cannot show the crystallization peak during both nonisothermal crystallization and the second heating procedure, the melting peak almost cannot be observed in the second heating process, whereas all PLLA/SrP samples have the obvious melting peaks, and the PLLA/1\% SrP exhibits the highest melting temperature $146.9^{\circ} \mathrm{C}$. Meantime, the higher cooling rate (above $5^{\circ} \mathrm{C} / \mathrm{min}$ ) cannot make PLLA form crystal (see Figure 4), resulting from the competition relevance between accelerated crystallization of $\mathrm{SrP}$ and retarded crystallization of cooling rate.

The different nonisothermal crystallization behavior will directly affect the glass transition temperature $\left(T_{g}\right)$ in the second heating procedure. Figure 5 shows $T_{g}$ of the pristine PLLA and PLLA containing $1 \mathrm{wt} \% \mathrm{SrP}$ at a heating rate of $10^{\circ} \mathrm{C} /$ min after nonisothermal crystallization. It is observed that PLLA/1\% SrP presents higher $T_{g}$ than the pristine PLLA. The reason is that the incorporation of SrP makes PLLA form more regular structure in cooling crystallization, and these regular structures firstly need to be destroyed before the glass transition of PLLA occurs, resulting in the increase of $T_{g}$ from $56.5^{\circ} \mathrm{C}$ to $59.6^{\circ} \mathrm{C}$. The similar result also can be observed in PLLA $/ N, N, N^{\prime}$-tris $(1 \mathrm{H}$-benzotriazole) trimesinic acid acethydrazide system [10].

3.3. XRD Analysis. XRD is the other important measurement method to evaluate the crystallization behavior of semicrystalline polymer. Usually, the higher the crystallinity is, the more intensity the diffraction peak exhibits. Figure 6 is the XRD curves of the pristine PLLA and PLLA/SrP after melt processed procedure. It is obvious that the intensity of the 


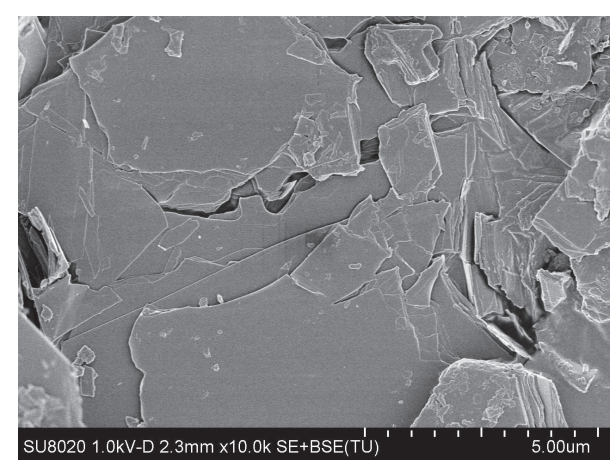

(a)

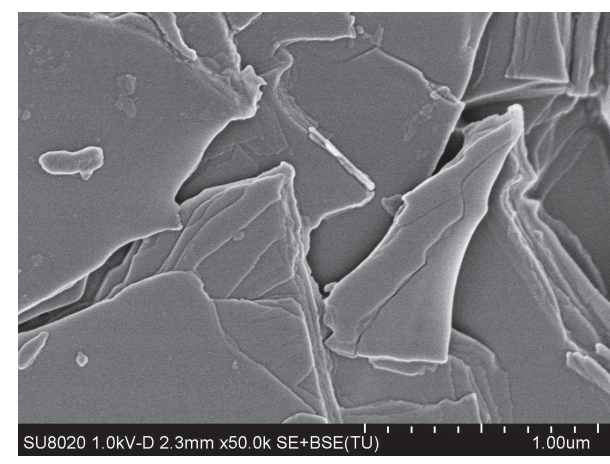

(c)

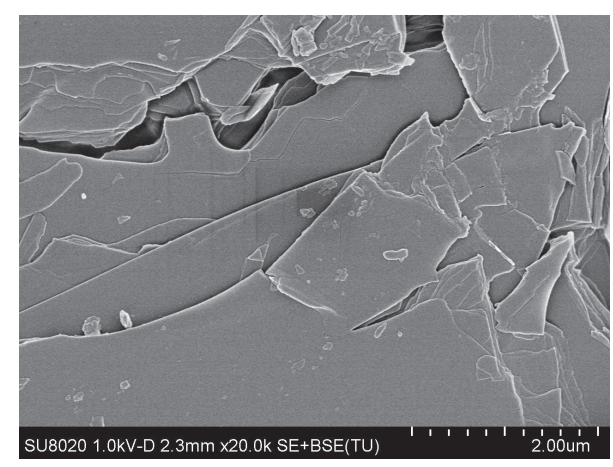

(b)

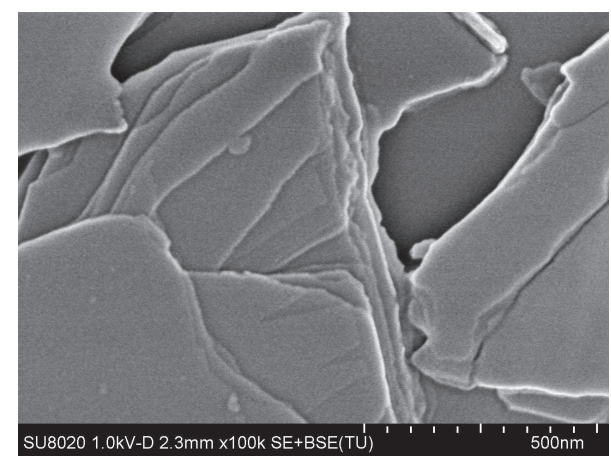

(d)

FIGURE 2: SEM of SrP.

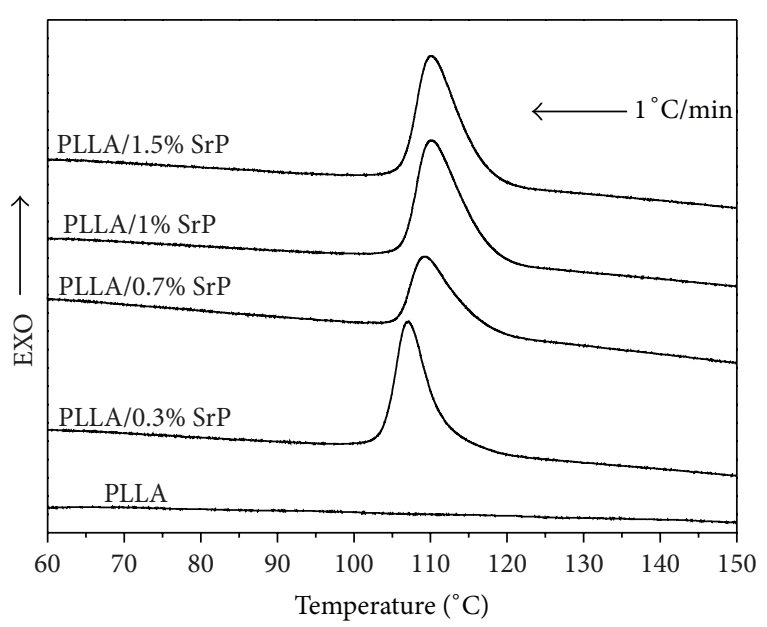

Figure 3: DSC curves of PLLA and PLLA/SrP samples at a cooling rate of $1^{\circ} \mathrm{C} / \mathrm{min}$.

characteristic diffraction peak of SrP gradually increases with the increase of SrP concentration in PLLA matrix, but PLLA and PLLA/SrP still exhibit the flat and broad diffraction peaks; the possible reason is that a small amount of crystal of PLLA or PLLA/SrP could not form at quite high cooling rate, and the cooling rate is the key factor determining the crystallization behavior of PLLA under this condition.

However, PLLA/SrP samples exhibit different XRD curves after nonisothermal crystallization at a cooling rate of

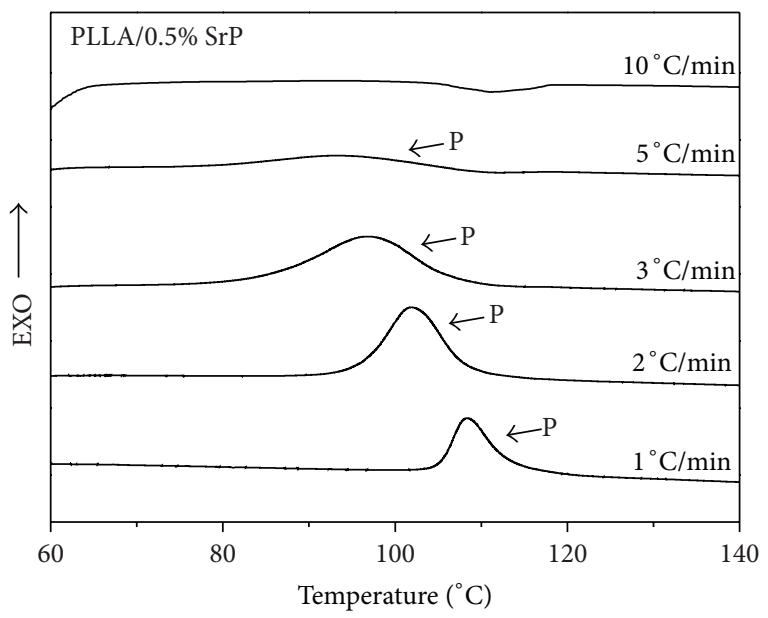

FIGURE 4: DSC curves of PLLA/0.5\% SrP at the different cooling rate.

$1^{\circ} \mathrm{C} / \mathrm{min}$ (see Figure 7). Though the diffraction peak of the pristine PLLA is still flat and broad, the diffraction peaks of PLLA/SrP samples become very sharp, and the characteristic diffraction peaks from the (110) plane and (203) plane appear at $2 \theta=16.7^{\circ}$ and $19.0^{\circ}$, respectively [22]; this difference further evidenced that $\mathrm{SrP}$ is very effective crystallization accelerator. Similarly, the intensity of diffraction peak of $\mathrm{SrP}$ strengthens with the increase of $\mathrm{SrP}$ concentration, but PLLA/SrP samples do not exhibit the regular change, and the PLLA $/ 1.5 \%$ SrP has the weakest diffraction peak 


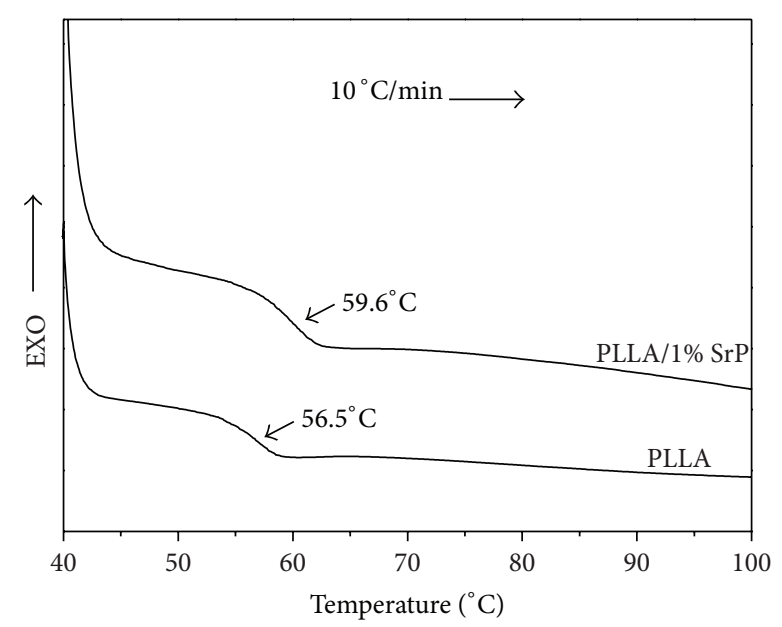

Figure 5: $T_{g}$ of PLLA and PLLA/1\% SrP after nonisothermal crystallization.

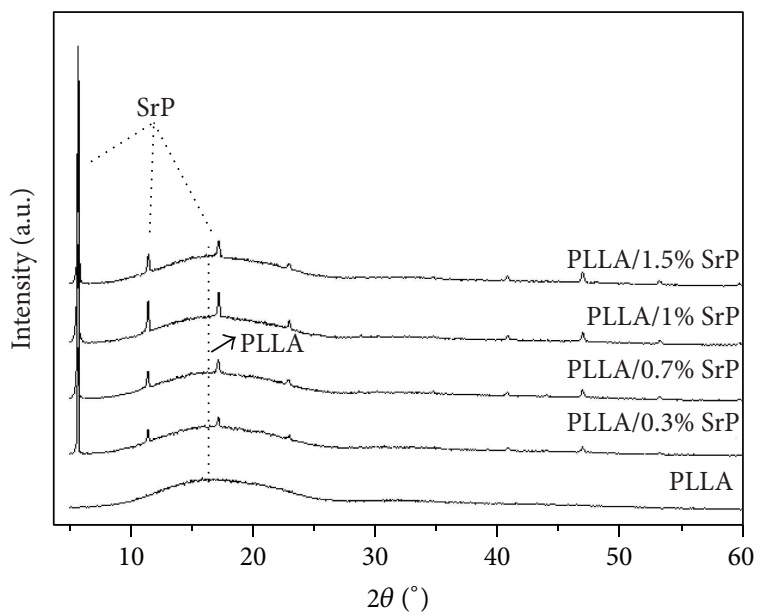

FIgURE 6: XRD curves of PLLA and PLLA/SrP after melting blend.

compared with other PLLA/SrP samples, indicating that excess $\mathrm{SrP}$ is a powerful inhibitor for PLLA crystallization. In contrast, PLLA $/ 0.7 \%$ SrP and PLLA/1\% SrP exhibit the stronger diffraction peak, showing that $0.7 \mathrm{wt} \%$ to $1 \mathrm{wt} \%$ is the optimum concentration range to achieve advanced nucleating effect.

3.4. Thermal Stability. Thermal stability is the key research index to achieve and broaden practical application. Figure 8 is the TGA curves of SrP, PLLA, and PLLA/SrP at the heating rate of $5^{\circ} \mathrm{C} / \mathrm{min}$ under $60 \mathrm{~mL} / \mathrm{min}$ air. As seen in Figure 8, the onset thermal decomposition temperature of $\mathrm{SrP}$ is much higher than that of PLLA and PLLA/SrP, showing that SrP has good thermal stability during melting blend of PLLA with $\mathrm{SrP}$, which is one of the prerequisites for SrP to serve as a nucleating agent for PLLA. And the onset decomposition temperatures of PLLA, PLLA/0.1\% SrP, PLLA/0.3\% SrP, PLLA/0.7\% SrP, PLLA/1\% SrP, and PLLA/1.5\% SrP are $341.1^{\circ} \mathrm{C}, 336.2^{\circ} \mathrm{C}, 334.2^{\circ} \mathrm{C}, 334.7^{\circ} \mathrm{C}, 335.3^{\circ} \mathrm{C}$, and $335.4^{\circ} \mathrm{C}$, respectively, showing that the introduction of $\mathrm{SrP}$ causes the

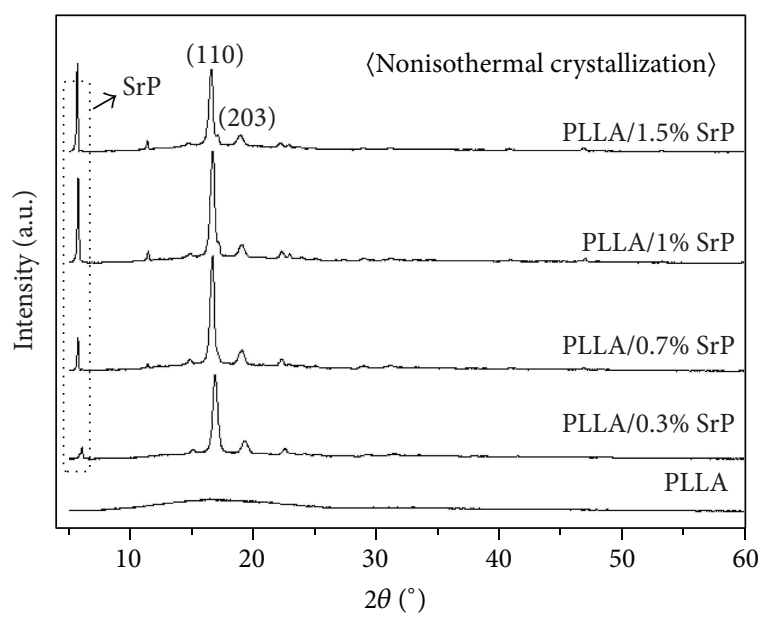

FIGURE 7: XRD curves of PLLA and PLLA/SrP after nonisothermal crystallization.

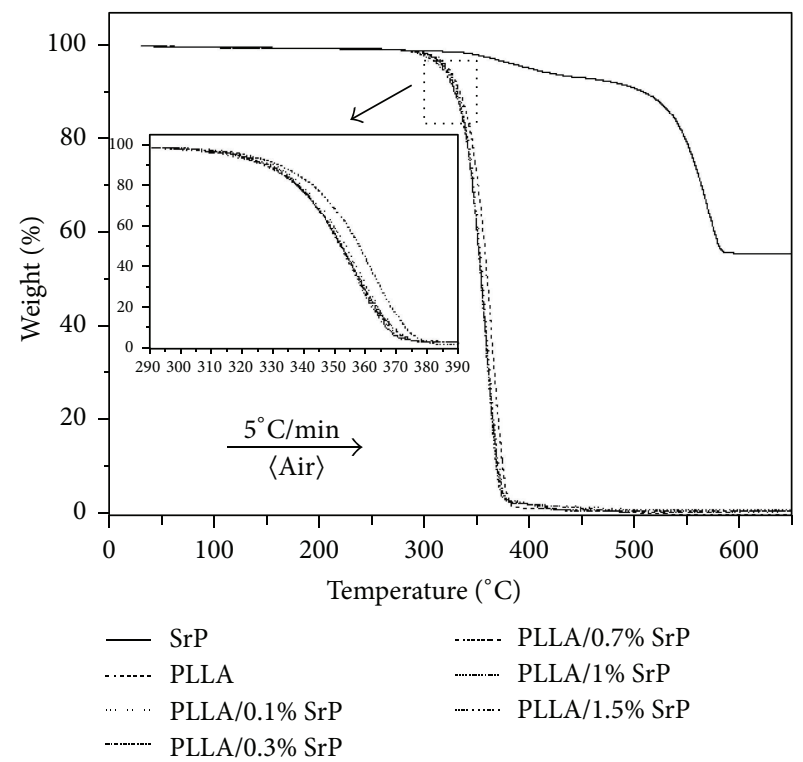

Figure 8: TGA curves of SrP, PLLA, and PLLA/SrP.

onset decomposition temperature of the PLLA to decrease. Moreover, apart from $0.1 \% \mathrm{SrP}$, the SrP concentration has no discernible effect on the onset thermal decomposition temperature of the PLLA. In addition, PLLA/SrP, as PLLA, exhibits one stage during thermal decomposition, indicating the relevant SrP concentration cannot change the thermal decomposition behavior of the pure PLLA.

\section{Conclusion}

Layered SrP was synthesized to investigate its influence on the nonisothermal crystallization and thermal stability of PLLA. The relevant results showed that layered SrP could serve as a crystallization accelerated agent for PLLA, and compared to the neat PLLA, $1 \mathrm{wt} \% \mathrm{SrP}$ made the $T_{o}$ and $T_{\mathrm{cp}}$ increase from $101.4^{\circ} \mathrm{C}$ and $94.5^{\circ} \mathrm{C}$ to $117.1^{\circ} \mathrm{C}$ and $110.1^{\circ} \mathrm{C}$, respectively, 
and $0.7 \mathrm{wt} \% \mathrm{SrP}$ caused $\Delta H_{c}$ to give rise to an increase from $0.1 \mathrm{~J} \cdot \mathrm{g}^{-1}$ to $31.0 \mathrm{~J} \cdot \mathrm{g}^{-1}$. The increase of $T_{g}$ and difference of XRD curves also further confirmed the enhancement effect of SrP to PLLA crystallization. In addition, the cooling rate could significantly affect the crystallization behavior of PLLA. Thermal decomposition showed that the addition of SrP could decrease the onset decomposition temperature of the pure PLLA, but the influence of SrP concentration on the thermal decomposition behavior of PLLA is negligible.

\section{Competing Interests}

The authors declare that there are no competing interests regarding the publication of this paper.

\section{Acknowledgments}

This work was supported by National Natural Science Foundation of China (Project no. 51403027), Natural Science Foundation of Chongqing Municipal Science and Technology Commission (Project no. cstc2015jcyjBX0123), and Scientific and Technological Research Program of Chongqing Municipal Education Commission (Project no. KJ131202).

\section{References}

[1] T. Xu, Y. M. Wang, D. R. He, Y. M. Xu, Q. Li, and C. Y. Shen, "Nucleation effect of layered metal phosphonate on crystallization of isotactic polypropylene," Polymer Testing, vol. 34, pp. 131-139, 2014.

[2] L. Beneš, K. Melánová, J. Svoboda, and V. Zima, "Intercalation behavior of calcium phenylphosphonate dihydrate $\mathrm{CaC}_{6} \mathrm{H}_{5} \mathrm{PO}_{3} \cdot 2 \mathrm{H}_{2} \mathrm{O}$," Journal of Inclusion Phenomena and Macrocyclic Chemistry, vol. 66, no. 3, pp. 279-284, 2010.

[3] L. Q. Zhang, X. Shi, S. M. Liu, V. K. Pareek, and J. Liu, "Organicinorganic hybrid hierarchical aluminum phenylphosphonate microspheres," Journal of Colloid and Interface Science, vol. 427, pp. 35-41, 2014.

[4] J. E. Haky, J. B. Brady, N. Dando, and D. Weaver, "Synthesis and structural studies of layered aluminum phenylphosphonate," Materials Research Bulletin, vol. 32, no. 3, pp. 297-303, 1997.

[5] A. H. Mahmoudkhani and V. Langer, "Layered calcium phenylphosphonate: synthesis and properties," Solid State Sciences, vol. 3, no. 4, pp. 519-525, 2001.

[6] F. Yu, P. J. Pan, N. Nakamura, and Y. Inoue, "Nucleation effect of layered metal phosphonate on crystallization of bacterial poly[(3-hydroxybutyrate)-co-(3-hydroxyhexanoate)]," Macromolecular Materials and Engineering, vol. 296, no. 2, pp. 103-112, 2011.

[7] T. Tsuboi, H. Katayama, and T. Itoh, "Crystallization behavior of poly(vinylidene fluoride) composites containing zinc phenylphosphonate," Polymer Engineering and Science, vol. 53, no. 4, pp. 843-848, 2013.

[8] Y. Z. Cai, Z. H. Guo, Z. P. Fang, and Z. H. Cao, "Effects of layered lanthanum phenylphosphonate on flame retardancy of glassfiber reinforced poly(ethylene terephthalate) nanocomposites," Applied Clay Science, vol. 77-78, pp. 10-17, 2013.

[9] S. He, X. Liu, H. Y. Zhao, Y. Zhu, and F. Z. Zhang, "Zirconium phenylphosphonate-anchored methyltrioxorhenium as novel heterogeneous catalyst for epoxidation of cyclohexene," Journal of Colloid and Interface Science, vol. 437, pp. 58-64, 2015.

[10] Y.-H. Cai, Y. Tang, and L.-S. Zhao, "Poly(l-lactic acid) with the organic nucleating agent $N, N, N^{\prime}$-tris( $1 H$-benzotriazole) trimesinic acid acethydrazide: crystallization and melting behavior," Journal of Applied Polymer Science, vol. 132, no. 32, Article ID 42402, 2015.

[11] P. Pan, J. Yang, G. Shan, Y. Bao, Z. Weng, and Y. Inoue, "Nucleation effects of nucleobases on the crystallization kinetics of poly(L-lactide)," Macromolecular Materials and Engineering, vol. 297, no. 7, pp. 670-679, 2012.

[12] Y. Xu and L. Wu, "Synthesis of organic bisurea compounds and their roles as crystallization nucleating agents of poly(l-lactic acid)," European Polymer Journal, vol. 49, no. 4, pp. 865-872, 2013.

[13] G.-X. Zou, Q.-W. Jiao, X. Zhang, C.-X. Zhao, and J.-C. Li, "Crystallization behavior and morphology of poly(lactic acid) with a novel nucleating agent," Journal of Applied Polymer Science, vol. 132, no. 5, Article ID 41367, 2015.

[14] S. Y. Xin, Y. Li, H. W. Zhao et al., "Confinement crystallization of poly(l-lactide) induced by multiwalled carbon nanotubes and graphene nanosheets: a comparative study," Journal of Thermal Analysis and Calorimetry, vol. 122, no. 1, pp. 379-391, 2015.

[15] Y. H. Cai, L. P. Ren, and Y. Tang, "Improvement of thermal properties of poly (L-lactic acid) by blending with zinc lactate," Journal of the National Science Foundation of Sri Lanka, vol. 43, no. 3, pp. 247-252, 2015.

[16] L. Han, P. Pan, G. Shan, and Y. Bao, "Stereocomplex crystallization of high-molecular-weight poly(l-lactic acid)/poly(dlactic acid) racemic blends promoted by a selective nucleator," Polymer, vol. 63, pp. 144-153, 2015.

[17] P. P. Pan, Z. C. Liang, A. Cao, and Y. Inoue, "Layered metal phosphonate reinforced poly(L-lactide) composites with a highly enhanced crystallization rate," ACS Applied Materials \& Interfaces, vol. 1, no. 2, pp. 402-411, 2009.

[18] S. S. Wang, C. Y. Han, J. J. Bian, L. J. Han, X. M. Wang, and L. S. Dong, "Morphology, crystallization and enzymatic hydrolysis of poly(L-lactide) nucleated using layered metal phosphonates," Polymer International, vol. 60, no. 2, pp. 284-295, 2011.

[19] V. Zima, J. Svoboda, L. Beneš, K. Melánová, and M. Trchová, "New strontium phenylphosphonate: synthesis and characterization," Solid State Sciences, vol. 8, no. 11, pp. 1380-1385, 2006.

[20] A. M. Lazarin and C. Airoldi, "Layered crystalline barium phenylphosphonate as host support for n-alkylmonoamine intercalation," Journal of Inclusion Phenomena, vol. 51, no. 1, pp. 33-40, 2005.

[21] B. Yang, Polylactic Acid, Chemical Industry Press, Beijing, China, 2007.

[22] Y. H. Cai, L. S. Zhao, and Y. H. Zhang, "Role of $N, N^{\prime}$ Bis( $1 H$-Benzotriazole) adipic acid acethydrazide in crystallization nucleating effect and melting behavior of poly(L-lactic acid)," Journal of Polymer Research, vol. 22, article 246, 2015. 

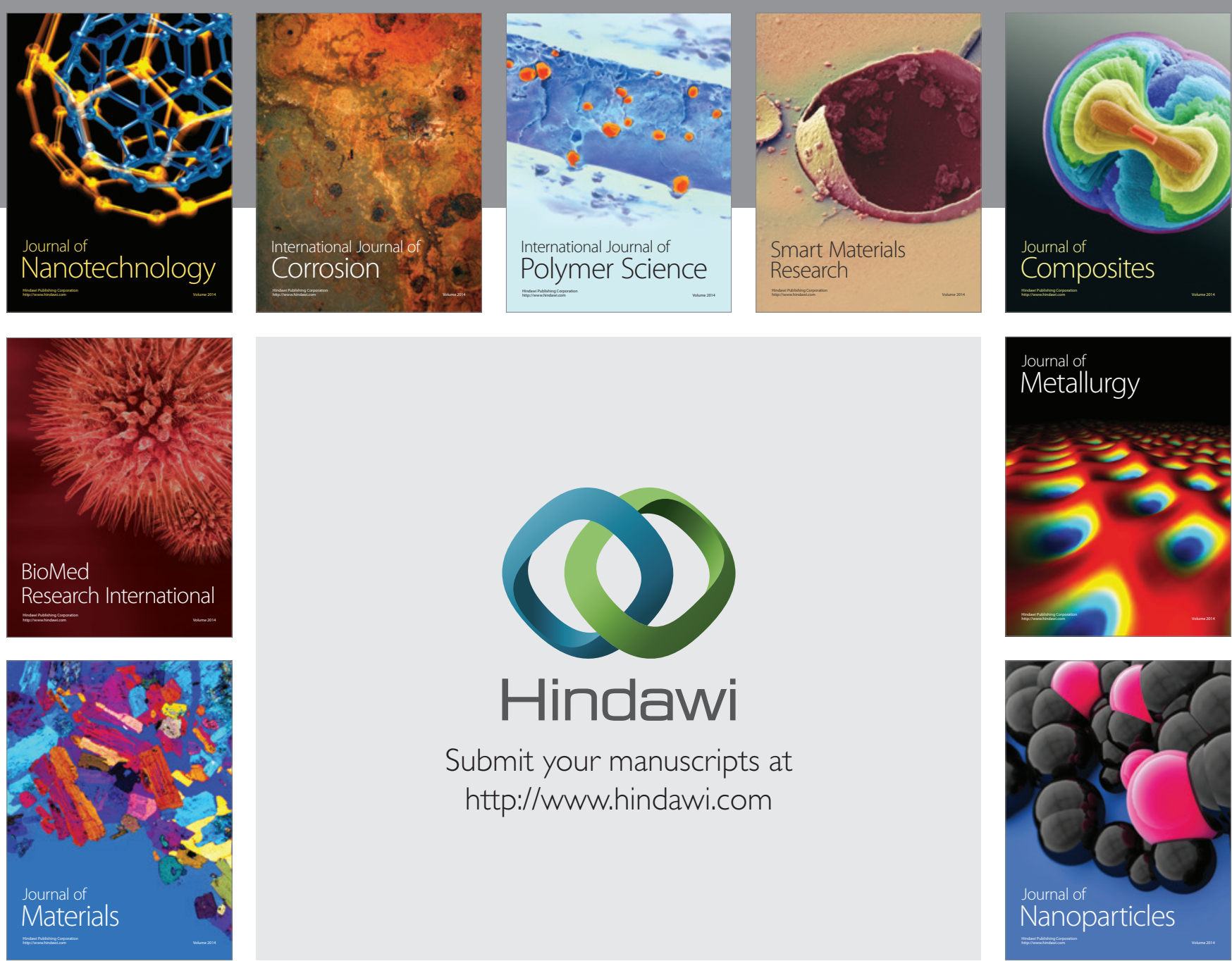

\section{Hindawi}

Submit your manuscripts at

http://www.hindawi.com

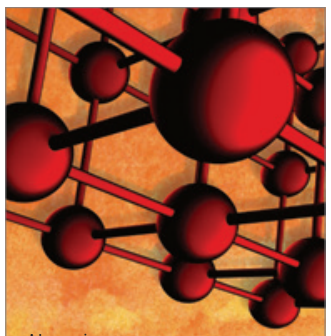

Materials Science and Engineering
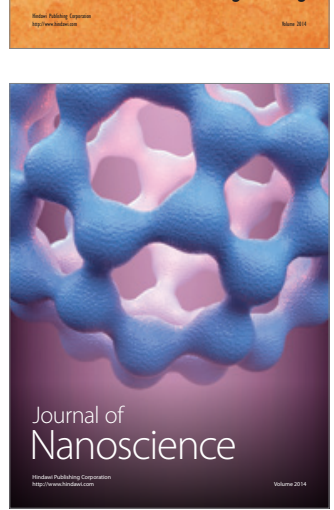
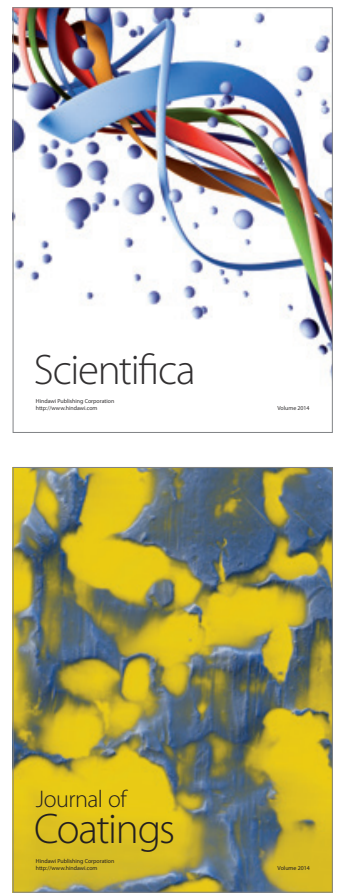
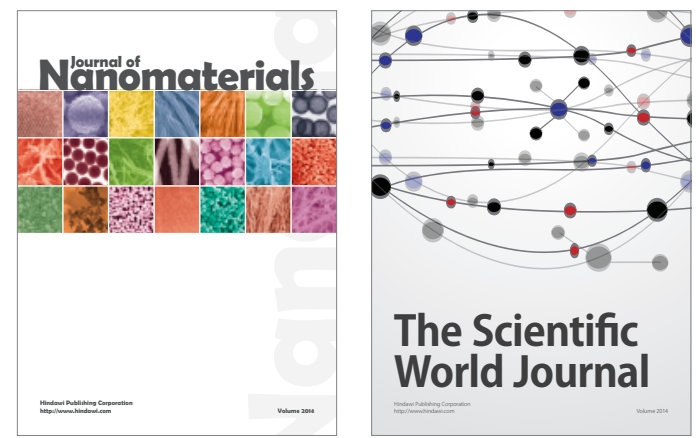

The Scientific World Journal
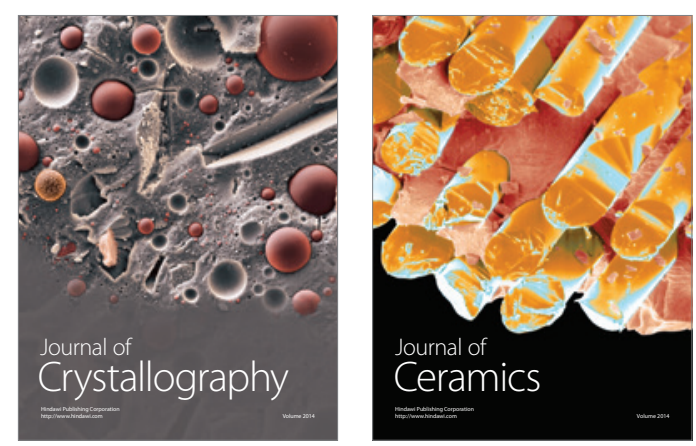
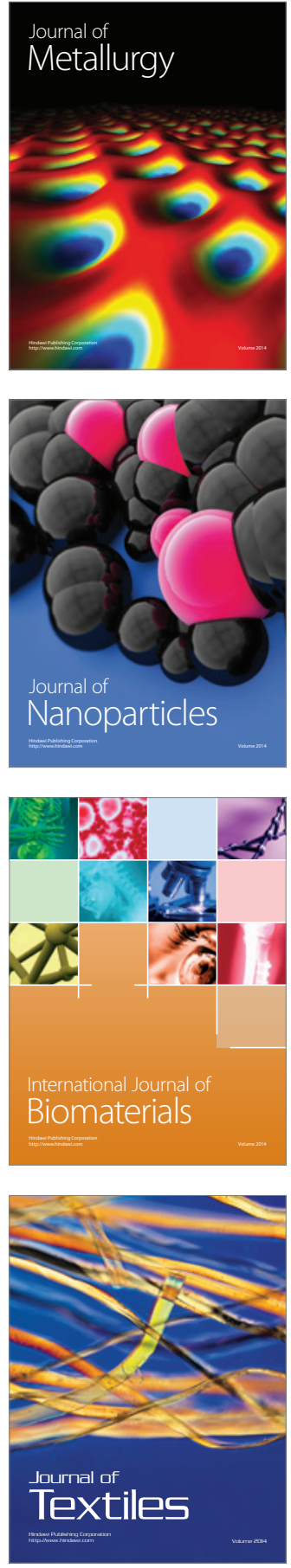